\title{
Resazurin Assay Data for Mycobacterium tuberculosis Supporting a Model of the Growth Accelerated by a Stochastic Non-Homogeneity
}

\author{
Eugene B. Postnikov ${ }^{1, * \mathbb{D}}$, Andrey A. Khalin ${ }^{1}$, Anastasia I. Lavrova ${ }^{2,3}$ and Olga A. Manicheva ${ }^{3}$ \\ 1 Department of Theoretical Physics, Kursk State University, Radishcheva st. 33, 305000 Kursk, Russia; \\ khalin_andrey@rambler.ru \\ 2 Medical Faculty, Saint-Petersburg State University, 21-thLine V.O. 8a, 199106 Saint-Petersburg, Russia; \\ aurebours@googlemail.com \\ 3 Saint-Petersburg State Research Institute of Phthisiopulmonology, Lygovsky Avenue 2-4, \\ 191036 Saint-Petersburg, Russia; olgamanicheva@rambler.ru \\ * Correspondence: postnicov@gmail.com; Tel.: +7-4712-51-0469
}

Received: 8 February 2019; Accepted: 25 February 2019; Published: 28 February 2019

\begin{abstract}
Tuberculosis is one of the most widespread worldwide diseases heavily affecting society. Among popular modern laboratory tests for mycobacterial growth, the resazurin assay has certain advantages due to its effectiveness and relatively low cost. However, the high heterogeneity of the mycobacterial population affects the average growth rate. This fact must be taken into account in a quantitative interpretation of these tests' output-fluorescence growth curves-related to the population growth of viable mycobacteria. Here, we report the spectrophotometric data obtained via the resazurin assay for the standard reference strain of Mycobacterium tuberculosis $\mathrm{H} 37 \mathrm{Rv}$ for different initial dilutions and generation numbers of the culture, as well as their primary processing from the point of view of the stochastic multiplicative growth model. The obtained data, which indicate an accelerated (instead of linear) growth of the population density logarithm between the end of the lag phase and the saturation, provide evidence of the importance of the growth rates' stochasticity. An analysis of the curve fits resulted in an estimation of the first two moments of the growth rates' probability distributions, showing its relevance to vital processes for mycobacterial culture.
\end{abstract}

Dataset: The full dataset of spectrophotometric data (fluorescence for each well of a platelet vs. the platelet's number and different initial conditions) is published as Supplementary Material to this paper in the journal Data.

\section{Dataset License: CC-BY-NC}

Keywords: Mycobacterium tuberculosis; stochastic growth rates; population growth models; accelerated growth

\section{Summary}

Tuberculosis (TB) remains one of the most widespread causes of death worldwide: around 10 million people around the world suffered from the developed TB disease in 2017 according to the current World Health Organization report [1]. The goal of fighting this illness requires intensive laboratory investigations of the population growth of its main infectious agent, Mycobacterium tuberculosis, as clinical samples, as well as in the context of the influence of anti-TB drugs. The latter is also quite important due to the emergence of multidrug-resistant TB [1]. Simultaneously, one needs relevant mathematical models aimed not only at fitting growth data obtained in vitro, 
but also at connecting the respective growth functions and coefficients (e.g., the growth rate) with conditions characterizing a bacterial community and a medium where the growth occurs [2]. Due to a complexity of factors affecting this growth, the development of such models is still active in the general bacterial case, as well as for mycobacterial growth, in particular, as described in [3-6]. In addition, there exists a demand on the experimental test datasets obtained in vitro of using clear standard mycobacterial cultures, which allows for testing such models and the premises of the growth mechanisms. The obtained data, which indicate an accelerated (instead of linear) growth of the population density logarithm between the end of the lag phase and the saturation, provide evidence of the importance of the growth rates' stochasticity. An analysis of the curve fits resulted in an estimation of the first two moments of the growth rates' probability distributions, showing its relevance to vital processes for mycobacterial culture.

Among the most popular modern methods of the express diagnostics of the mycobacterial growth, one can note the resazurin (also known as the Alamar Blue dye) assay based on the oxidation-reduction process leading to the color change of the blue non-fluorescent resazurin to highly fluorescent pink resorufin due to bacterial metabolism of viable cells [7-11], i.e., it allows for estimating bacterial count, as well as taking into account physiological processes accompanying and controlling the multiplication of bacteria.

With respect o M. tuberculosis, it should be pointed out that recent studies have indicated a high variability in the individual cells' proliferation and growth rates even within a population of a uniform strain culture [12-14]. This may induce deviations from the conventional behavior of bacterial growth curves, which normally include the lag-phase, the exponential growth phase, the saturation phase, and the decay phase, and, with respect to two intermediate phases in the aforementioned list, be modeled by the classic population growth models like Verhulst, Gompertz, Richards, etc. Some examples are shown in Figure 1B.

Note that such models imply the existence of the exponential growth phase, i.e., the linearity of the population density $N$ reduced to its value at the phase's beginning $N_{0}$ in semi-logarithmic co-ordinates $\log \left(N / N_{0}\right)=r t$, where $r$ is the growth rate. In a general case, there are concave up curves tending to the saturation due to a finite medium carrying capacity.

On the contrary, it has been recently revealed [15] that the stochasticity in the growth rates, under quite general assumptions on their probability density distribution, results in the replacement of the semilogarithmic linearity with the quadratic logarithmic growth function $\log \left(N / N_{0}\right)=r t+\left(\sigma^{2} / 2\right) t^{2}$. Namely, a similar curve shape is detected in the experimental observations in Figure 1A, and the details will be given in the section Data Description.

Thus, in order to investigate these stochastic effects on the growth curves of a mycobacterial population, we report a detailed study of the mycobacterial growth curves spectrophotometrically detected via the resazurin assay microtiter kit in the case of a variety of sample preparation conditions. All measurements reported are evaluated with the strain H37Rv of Mycobacterium tuberculosis, which is a standard reference strain in TB research [16].

\section{Data Description}

The dataset provided in this paper presents the dynamics of fluorescence activity recorded during the resazurin assay test with the strain H37Rv of M. tuberculosis taken from the strain library maintained by the Saint-Petersburg State Research Institute of Phthisiopulmonology acting under the Ministry of Health of the Russian Federation.

The details of samples' preparation and measurement procedures evaluated under different initial conditions are given in the section "Method" below. The principal groups of data are listed in Table 1. They represent four possible combinations of initial conditions for the mycobacterial cultures: two different initial dilutions determined the initial population density (found optically before placing the culture into the microplate kit) and two different generations cultured previously. To avoid possible bias, the data presented in Columns 1-8, 9-16, and 17-20 in the Supplementary Data 
(the sheet "Raw data"), and referred to as "sample \#" in the plots and discussions below, originate from experiments separated by two-month intervals between them; their initial seeds were prepared separately as well.

Table 1. Experimental (initial concentration (I.c.) and generation's number (I.g.) characteristics of the mycobacterial sample groups, as well as the characteristics of their population growth curves as indicated by the spectrophotometric data for the first 4 days (the accelerating phase of the growth) supplied with the standard errors (S.E.).

\begin{tabular}{lcccccc}
\hline & I.c./(cfu/mL) & I.g. & $\langle\boldsymbol{r}\rangle /$ days $^{-1}$ & S.E./days & $\langle\sigma\rangle /$ days $^{-1}$ & S.E./days $^{-1}$ \\
\hline Group 1 & $1.5 \cdot 10^{7}$ & 2 & 0.32 & 0.02 & 0.34 & 0.02 \\
Group 2 & $7.5 \cdot 10^{6}$ & 2 & 0.18 & 0.01 & 0.42 & 0.02 \\
Group 3 & $1.5 \cdot 10^{7}$ & 7 & 0.22 & 0.01 & 0.39 & 0.02 \\
Group 4 & $7.5 \cdot 10^{6}$ & 7 & 0.20 & 0.02 & 0.37 & 0.02 \\
\hline
\end{tabular}

\section{Data Processing and Analysis}

Figure 1A shows typical data of fluorescence dynamics for all four groups. The data points denoted via markers were obtained as values averaged over eight individual wells of the microplate's row. Since the measurement procedure was designed to start recordings after the lag-phase, the growth phase, the saturation phase, and the decay phase are visible. The time points for all data are depicted in the semi-logarithmic scale and normalized with respect to the first point. This visibly differs from the classic saturating growth shown in Figure 1B. Namely, they are concave down up to the time moments very close to the saturation. This confirms that this behavior cannot be explained by the lag-phase effects and should be considered as the consequence of an existence of additional mechanisms affecting the active bacterial growth phase.

To reveal the respective growth law, we fitted five first data points for each sample (they were chosen as corresponding to the active growth phase, which does not reach the saturation guaranteed) by the quadratic polynomial using the standard MATLAB/OCTAVE function polyfit, which calculates the best fit in a least-squares sense:

$$
\log \left(\frac{N}{N_{0}}\right)=p_{2} t^{2}+p_{1} t+p_{0}=r t+\frac{\sigma^{2}}{2} t^{2},
$$

where $p_{j}, j=2, \ldots, 0$ are the polynomial coefficients. The value of the constant term $p_{0}$ shows a deviation of the logarithm of initial population $N(0)$ provided by the fit from its actual value $N_{0}$; in fact, it did not deviate from zero within the accuracy of data and, therefore, was negligible for all samples. The coefficient $p_{1}$ takes into account the exponential component of the population growth function $N(t)$; it is the leading term in the case of normal Malthusian growth of a uniform population and represents the average Malthusian growth rate otherwise. For this reason, we denoted it as $p_{1}=r$ for uniformity with the stochastic growth model discussed below. This model argues that the distribution of growth rates leads to an emergence of the quadratic component with the rate $p_{1}$ connected to the variance of the growth rates, i.e., the notation $p_{2}=\sigma^{2} / 2$ is introduced.

One can see in Figure 1A that these second-order polynomials satisfy the experimental data with high accuracy, the average correlation coefficient for all samples being equal to 0.99 and the standard deviation to 0.014 . Thus, we can conclude that, in addition to the classic uniform average Malthusian growth (the term $r t$ in Equation (1)), there exists an additional term $\sigma^{2} t^{2} / 2$ describing an accelerated growth (it is positive for all samples, $p_{2}>0$ ) far from the saturation conditions, but sufficiently after the lag phase. 

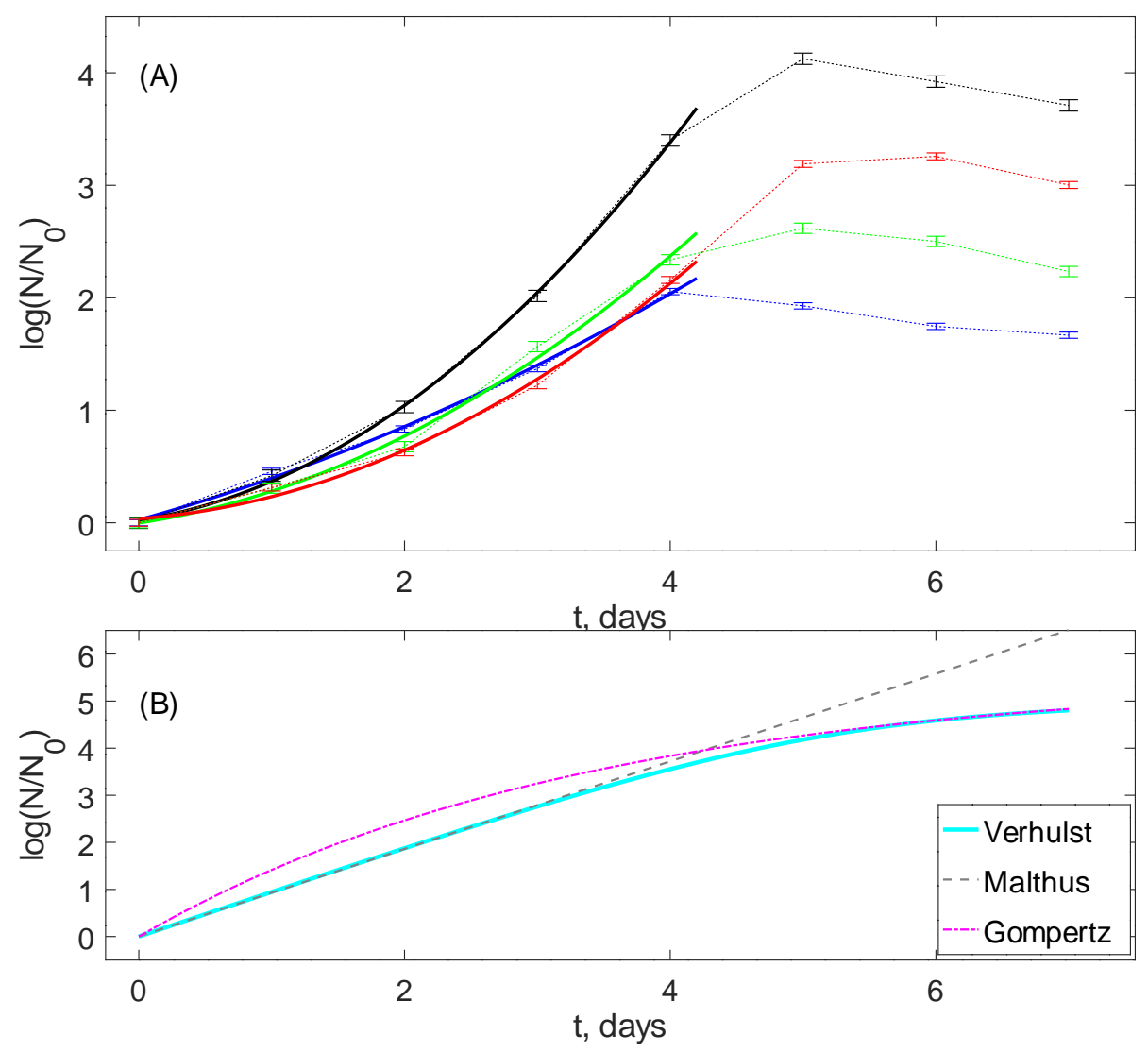

Figure 1. (A) Examples of experimental data (logarithms of fluorescence intensity normalized with respect to the initial value), one for each of the groups listed in Table 1 shown as markers with standard errors denoted (blue: Sample \#17; black: Sample \#14; green: Sample \#7; red: Sample \#8); numbers are given with respect to the Supplementary Data file; dotted lines connect the markers for the sake of visibility only, as well as parabolic curves fitting them during the first four days of observations. (B) Standard population growth curves in the same semi-logarithmic normalized representation as in (A), which demonstrates the opposite bending direction relative to the experimental data shown in (A).

The distribution of $r$ and $\sigma$ for all trials marked with respect to the samples groups is shown in Figure 2. One can see that the values of the average growth rates $r$ form a quite localized, bounded stripe with outliers with relatively larger values for Group $1 ; \sigma$ values are distributed more uniformly among all groups. This conclusion is supported by the numerical value averaged over each group presented in Table 1. For the first group, $\langle r\rangle$ was 1.5-times larger than for Groups 2-4, while the dispersion of $\langle\sigma\rangle$ was not so drastic. 

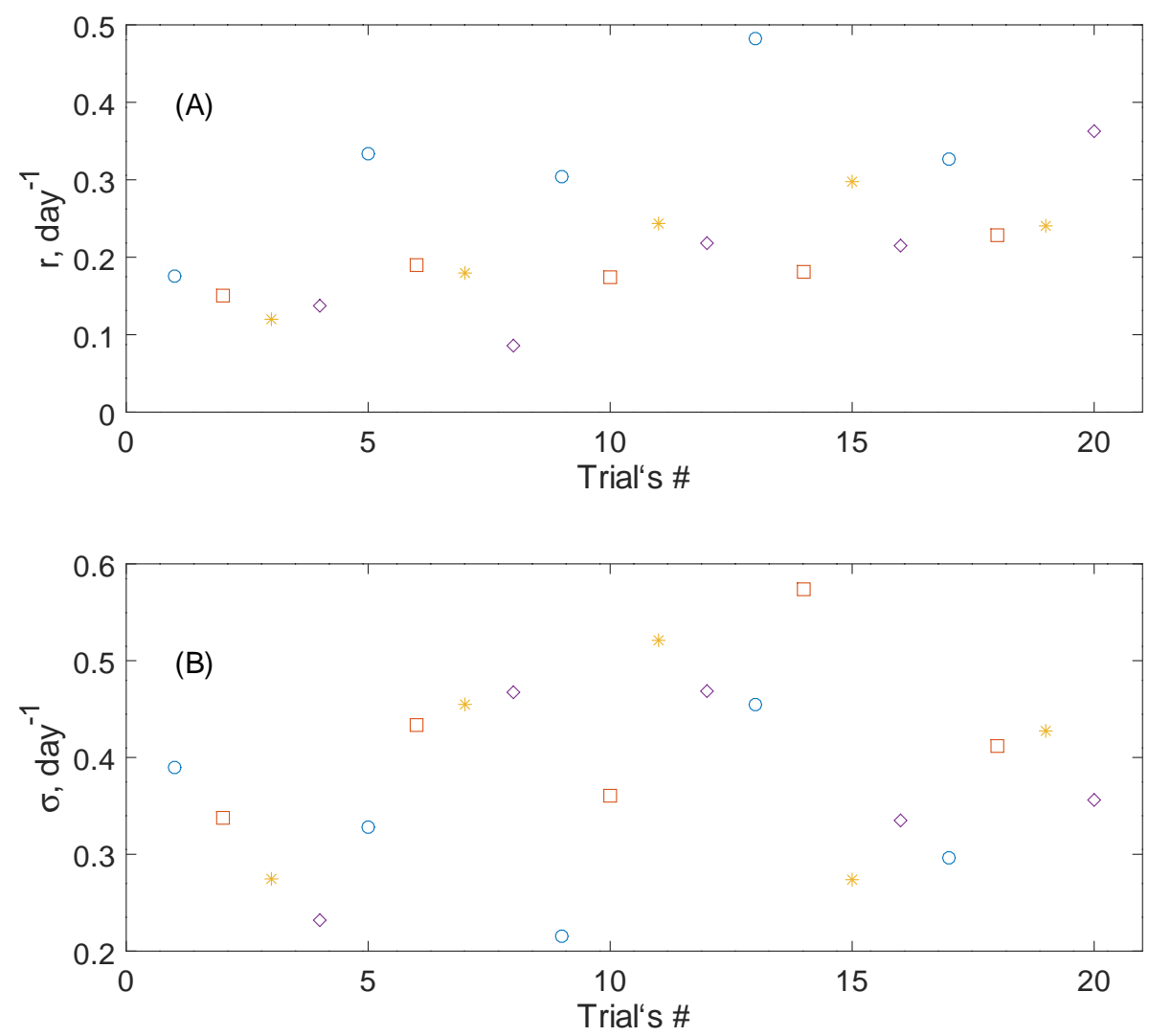

Figure 2. Growth rates (A) and dispersions (B) for each trial in the Supplementary Data file; circles, squares, asterisks, and diamonds correspond to Groups 1-4, respectively.

\section{Data Discussion with Respect to the Stochastic Growth Model}

Following [15], let us consider the growing mycobacterial culture as consisting of multiple sub-populations $N_{P}$ (individual bacteria, bacterial colonies, or even groups of colonies) parametrized by the variable $P$, each of which grows exponentially within the chosen time interval between the lag-phase and the start of saturation:

$$
\frac{d N_{P}}{d t}=k(P) N_{p} ; \quad N_{P}(t)=N_{0} e^{k(P) t},
$$

where the partial growth rate $k_{P}=$ const for each sub-population, and the initial value $N_{P}(t)=N_{0}$ is the same for all.

Assuming random variations of the individual growth rates, the observable quantity is the mean value for the whole population with respect to the probability density function $p(P)$ :

$$
N(t)=\left\langle N_{P}(P)\right\rangle=N_{0} \int_{\{P\}} e^{k(P) t} p(P) d P .
$$

For simplicity, let us consider the growth rates parametrized as $k(P)=P$ and take $p(P)$ as the Gaussian normal distribution with the mean $r$ and the dispersion $\sigma$. Then,

$$
N(t)=N_{0} \int_{-\infty}^{+\infty} e^{P t} e^{-\frac{(P-\mu)^{2}}{2 \sigma^{2}}} \frac{d P}{\sqrt{2 \pi \sigma^{2}}}=N_{0} e^{r t+\frac{\sigma^{2}}{2} t^{2}}
$$

Note that we have taken negative $k=P$, which corresponds to a possible presence of initial bacteria, which become extinct during the experiment. In fact, more complex dependencies of 
the growth rate on the substrate consumption described by more complicated functions $k(P)$, e.g., Jacob-Monod's (Holling II), as well as more complex probability distributions $p(P)$ observable in population dynamics, can be reduced to the same Gaussian-based form (2) within the considered characteristic time interval, introducing an effective mean growth rate $\mu$ and an effective acceleration $\sigma^{2}$; see [15] for details.

Comparing Equations (1) and (2), we can conclude that the revealed fit corresponded to the growth of a mixed population, where the mean duplication time $T=\log (2) /\langle\mu\rangle$ varied from $52 \mathrm{~h}$ (for Group 1) to $92 \mathrm{~h}$ (for Group 2). These values belong to the reasoned generation time for Mycobacterium tuberculosis, which can vary from 16-160 $\mathrm{h}$ in dependent of the internal (metabolic) or external (medium-based) conditions $[17,18]$. In addition, we stress that it is the mean value for the stochastic population with a wide dispersion of growth rates; compare $\langle\sigma\rangle$ and $\langle r\rangle$, which are similar in value. Moreover, for larger initial generations and/or lower initial concentrations, $\langle\sigma\rangle\rangle\langle\mu\rangle$, which indicates even the presence of dying bacteria. On the other hand, the upper limit of generation times within the standard deviation $T_{0.68}=\log (2) /(\langle\sigma\rangle+\langle r\rangle)$ varied within 25 and $27 \mathrm{~h}$, and $T_{0.95} \log (2) /(\langle\sigma\rangle+2\langle r\rangle)$ lied within the more narrow interval of 16.3-17.2 $\mathrm{h}$ for the doubled standard deviation (subscripts 0.68 and 0.95 indicate the percentage bounds for the distributed population) that accurately corresponds to the known biological lower boundary for the mycobacterial duplication time.

\section{Method}

The principal protocol followed [19] with some adjustments to the actual conditions of the experiment. The suspension of $M$. tuberculosis was unfrozen for three weeks before the experiment and cultured on Löwenstein-Jensen medium. The suspension was prepared as follows: the bacterial culture was placed into a dry sterile tube together with 10 glass spheres (3-5 $\mathrm{mm}$ in diameter). The bacterial mass was rubbed with the shaker "Vortex", and 3-4 drops of the Middlebrook 7H9 broth (Becton-Dickinson; Franklin Lakes, NJ, USA) were added, then the system was rubbed again. Then, $5 \mathrm{~mL}$ of the Middlebrook 7H9 broth were added, and the system was kept for $1 \mathrm{~h}$ to precipitate conglomerates. The upper part of the supernatant $(2 \mathrm{~mL})$ was processed to obtain a density equal to 1 McFarland unit. The obtained suspension was 40-times diluted by the Middlebrook broth supplied with 10\% OADC (oleic acid, albumin, dextrose, and catalase) (Becton-Dickinson; Franklin Lakes, NJ, USA) and $0.01 \%$ water of resazurin ( $30 \mu \mathrm{L}$ for a $200-\mu \mathrm{L}$ volume prepared for distribution among the wells of the microtiter plate) and was inoculated into a 96-well plate.

Fluorescence was measured by the FLUOstar OPTIMA microplate-based multi-detection reader (www.bmglabtech.com) with the excitation and emission wavelengths of $530 \mathrm{~nm}$ and $590 \mathrm{~nm}$, respectively, during 7 days daily.

To process the obtained data and generate the numerical values given in Table 1 and the figures, the free scientific software GNU OCTAVE (https:/ /www.gnu.org/software/octave/) was used with its standard functions of statistical analysis and polynomial fitting. The Supplementary Material to this work contains the program code written for this goal, which directly used the data file in Excel format, provided there as well.

\section{Conclusions and Outlooks}

In this work, we presented a dataset that consists of the growth curves of the standard reference strain of Mycobacterium tuberculosis H37Rv obtained via the spectrophotometric resazurin assay, which exhibit a non-exponential shape even after completing the lag-phase. This phenomenon and the revealed growth parameters were interpreted as satisfying the recently proposed model of averaging over a non-homogenous population with the stochastic distribution of growth rates. This differs from the behavior of the accelerated growth during the lag-phase, where the reduplication of cells goes with one and the same growth rate, but starts from different time moments.

It should be pointed out that similar replacement of linear or concave down growth curves by the concave up one within the phase of an active growth may be traced back to the early 
stages of microbiological research [20], but for a long time, was not under consideration since the paradigmatic mathematical models of the average single population multiplication operates with uniform communities, which do not exhibit such behavior.

However, recently, such non-standard features started to attract the attention of the research community involved in an application of dynamical and stochastic systems theory for modeling macroscopic growth dynamics [21-24]. The key aim of such research is to connect the revealed genetic/metabolic variability of individual microorganisms with the macroscopic dynamics of the whole population. With respect to this area of research, Mycobacterium tuberculosis, which is known as having a relatively large variability range even within one standardized strain, seems to be a good prospective candidate as a model organism, and the reported data may play a role in the benchmarking for testing future, more detailed mathematical models of microbiological dynamics.

Supplementary Materials: The following are available online at http:/ / www.mdpi.com/2306-5729/4/1/36/s1, Excel file: spectrophotometric data; OCTAVE's m-file: the code used for data processing.

Author Contributions: E.B.P. and A.I.L. conceptualized the study and wrote the manuscript; E.B.P. revised the computer code and analyzed and fitted the data; A.I.L. analyzed and curated the output of data processing; A.A.K. wrote the primary computer code and evaluated the data preprocessing and primary analysis; O.A.M. performed the experiment; all authors revised the paper.

Funding: E.B.P., A.A.K., and A.I.L. are supported by the Ministry of Education and Science of the Russian Federation within the research Project No. 3.9499.2017/8.9.

Conflicts of Interest: The authors declare no conflict of interest. The funders had no role in the design of the study; in the collection, analyses, or interpretation of data; in the writing of the manuscript; nor in the decision to publish the results.

\section{References}

1. World Health Organization. Global Tuberculosis Report 2018; World Health Organization: Geneva, Switzerland, 2018.

2. Painter, P.R.; Marr, A.G. Mathematics of microbial populations. Annu. Rev. Microbiol. 1968, 22, 519-548. [CrossRef] [PubMed]

3. López, S.; Prieto, M.; Dijkstra, J.; Dhanoa, M.S.; France, J. Statistical evaluation of mathematical models for microbial growth. Int. J. Food Microbiol. 2004, 96, 289-300. [CrossRef] [PubMed]

4. Peleg, M.; Corradini, M.G. Microbial growth curves: What the models tell us and what they cannot. Crit. Rev. Food Sci. Nutr. 2011, 51, 917-945. [CrossRef] [PubMed]

5. Werlang, P.; Fagundes, M.Q.; Adamatti, D.F.; Machado, K.S.; von Groll, A.; da Silva, P.E.; Werhli, A.V. Multi-agent-based simulation of mycobacterium tuberculosis growth. In Proceedings of the International Workshop on Multi-Agent Systems and Agent-Based Simulation, Saint Paul, MN, USA, 6-7 May 2013; Springer: Berlin, Heidelberg, Germany, 2013; pp. 131-142.

6. Song, H.S.; Cannon, W.; Beliaev, A.; Konopka, A. Mathematical modeling of microbial community dynamics: A methodological review. Processes 2014, 2, 711-752. [CrossRef]

7. Yajko, D.M.; Madej, J.J.; Lancaster, M.V.; Sanders, C.A.; Cawthon, V.L.; Gee, B.; Babst, A.; Hadley, W.K. Colorimetric method for determining MICs of antimicrobial agents for Mycobacterium tuberculosis. J. Clin. Microbiol. 1995, 33, 2324-2327. [PubMed]

8. Collins, L.A.; Franzblau, S.G. Microplate alamar blue assay versus BACTEC 460 system for high-throughput screening of compounds against Mycobacterium tuberculosis and Mycobacterium avium. Antimicrob. Agents Chemother. 1997, 41, 1004-1009. [CrossRef] [PubMed]

9. Martin, A.; Portaels, F.; Palomino, J.C. Colorimetric redox-indicator methods for the rapid detection of multidrug resistance in Mycobacterium tuberculosis: A systematic review and meta-analysis. J. Antimicrob. Chemother. 2007, 59, 175-183. [CrossRef] [PubMed]

10. Rampersad, S.N. Multiple applications of Alamar Blue as an indicator of metabolic function and cellular health in cell viability bioassays. Sensors 2012, 12, 12347-12360. [CrossRef] [PubMed]

11. Präbst, K.; Engelhardt, H.; Ringgeler, S.; Hübner, H. Basic colorimetric proliferation assays: MTT, WST, and Resazurin. In Cell Viability Assays: Methods and Protocols; Gilbert, D.F., Friedrich, O., Eds.; Springer Science+Business Media LLC: New York, NY, USA, 2017; pp. 1-17. 
12. Aldridge, B.B.; Fernandez-Suarez, M.; Heller, D.; Ambravaneswaran, V.; Irimia, D.; Toner, M.; Fortune, S.M. Asymmetry and aging of mycobacterial cells lead to variable growth and antibiotic susceptibility. Science 2012, 335, 100-104. [CrossRef] [PubMed]

13. Kieser, K.J.; Rubin, E.J. How sisters grow apart: Mycobacterial growth and division. Nat. Rev. Microbiol. 2014, 12, 550-562. [CrossRef] [PubMed]

14. Logsdon, M.M.; Aldridge, B.B. Stable Regulation of Cell Cycle Events in Mycobacteria: Insights from Inherently Heterogeneous Bacterial Populations. Front. Microbiol. 2018, 9, 514. [CrossRef] [PubMed]

15. Khalin, A.A.; Postnikov, E.B.; Ryabov, A.B. Stochastic effects in mean-field population growth: The quasi-Gaussian approximation to the case of a Taylor's law-distributed substrate. Phys. A Stat. Mech. Appl. 2018, 511, 166-173. [CrossRef]

16. Cole, S.T.; Brosch, R.; Parkhill, J.; Garnier, T.; Churcher, C.; Harris, D.; Gordon, S.V.; Eiglmeier, K.; Gas, S.; Barry Iii, C.E.; et al. Deciphering the biology of Mycobacterium tuberculosis from the complete genome sequence. Nature 1998, 393, 537-544. [CrossRef] [PubMed]

17. Gill, W.P.; Harik, N.S.; Whiddon, M.R.; Liao, R.P.; Mittler, J.E.; Sherman, D.R. A replication clock for Mycobacterium tuberculosis. Nat. Med. 2009, 15, 211-214. [CrossRef] [PubMed]

18. Beste, D.J.V.; Espasa, M.; Bonde, B.; Kierzek, A.M.; Stewart, G.R.; McFadden, J. The genetic requirements for fast and slow growth in mycobacteria. PLoS ONE 2009, 4, e5349. [CrossRef] [PubMed]

19. Von Groll, A.; Martin, A.; Portaels, F.; da Silva, P.E.A.; Palomino, J.C. Growth kinetics of Mycobacterium tuberculosis measured by quantitative resazurin reduction assay: A tool for fitness studies. Braz. J. Microbiol. 2010, 41, 300-303. [CrossRef] [PubMed]

20. Lodge, R.M.; Hinshelwood, C.N. Physicochemical aspects of bacterial growth. Part VIII. Growth of Bacterium lactis aerogenesinmedia containing ammonium sulphate or various amino acids. J. Chem. Soc. 1943, 208-213. [CrossRef]

21. Pulkkinen, O.; Metzler, R. Variance-corrected Michaelis-Menten equation predicts transient rates of single-enzyme reactions and response times in bacterial gene-regulation. Sci. Rep. 2015, 5, 17820. [CrossRef] [PubMed]

22. Herrmann, H.; Lawless, C. Modeling the consequences of heterogeneity in microbial population dynamics. bioRxiv 2017, 124412. [CrossRef]

23. Schmitz, A.C.; Hartline, C.J.; Zhang, F. Engineering microbial metabolite dynamics and heterogeneity. Biotechnol. J. 2017, 12, 1700422. [CrossRef] [PubMed]

24. Allen, R.J.; Waclaw, B. Bacterial growth: A statistical physicist's guide. Rep. Prog. Phys. 2019, 82, 016601. [CrossRef] [PubMed]

(C) 2019 by the authors. Licensee MDPI, Basel, Switzerland. This article is an open access article distributed under the terms and conditions of the Creative Commons Attribution (CC BY) license (http://creativecommons.org/licenses/by/4.0/). 